\title{
Type I and type IV pili of Xylella fastidiosa affect twitching motility, biofilm formation and cell-cell aggregation
}

\author{
Yaxin Li, Guixia Hao, Cheryl D. Galvani, Yizhi Meng,† \\ Leonardo De La Fuente, H. C. Hoch and Thomas J. Burr

\begin{abstract}
Department of Plant Pathology, Cornell University - New York State Agricultural Experiment Station, Geneva, NY 14456, USA
\end{abstract}

Correspondence

Thomas J. Burr

tjb1@cornell.edu

Received 10 September 2006

Revised 13 November 2006

Accepted 14 November 2006

\begin{abstract}
Xylella fastidiosa, an important phytopathogenic bacterium, causes serious plant diseases including Pierce's disease of grapevine. It is reported here that type I and type IV pili of $X$. fastidiosa play different roles in twitching motility, biofilm formation and cell-cell aggregation. Type I pili are particularly important for biofilm formation and aggregation, whereas type IV pili are essential for motility, and also function in biofilm formation. Thirty twitching-defective mutants were generated with an EZ : :TN transposome system, and several type-IV-pilus-associated genes were identified, including fimT, pilX, pilY1, pilO and pilR. Mutations in fimT, pilX, pilO or pilR resulted in a twitch-minus phenotype, whereas the pilY1 mutant was twitching reduced. A mutation in fimA resulted in a biofilm-defective and twitching-enhanced phenotype. A fimA/pilO double mutant was twitch minus, and produced almost no visible biofilm. Transmission electron microscopy revealed that the pili, when present, were localized to one pole of the cell. Both type I and type IV pili were present in the wild-type isolate and the pilY1 mutant, whereas only type I pili were present in the twitch-minus mutants. The fimA mutant produced no type I pili. The fimA/pilO double mutant produced neither type I nor type IV pili.
\end{abstract}

\section{INTRODUCTION}

Xylella fastidiosa is a Gram-negative non-flagellated bacterium that is limited to colonizing the water-conducting xylem vessels of host plants. A number of economically important diseases are caused by the bacterium, including Pierce's disease of grapevine (Vitis vinifera L.), citrus variegated chlorosis, pear leaf scorch, and almond leaf scorch (Purcell, 1996, 1997). Disease symptoms are thought to occur as a result of water stress and nutritional deficiencies caused by blockage of the xylem vessels by the bacterium (Hopkins, 1989). The bacteria are transmitted to plants by xylem-feeding insect vectors, such as glassy-winged and blue-green sharpshooters (Hopkins, 1989; Tubajika et al., 2004; Hill \& Purcell, 1995). To date, the molecular mechanisms of virulence of $X$. fastidiosa, as well as how it interacts with plant hosts, have not been elucidated. Hopkins (1989) reported that colonization and pathogenicity of the Pierce's disease strains of $X$. fastidiosa are dependent on their ability to move within the xylem vessels, i.e. avirulent or weakly

†Present address: Department of Biomedical Engineering, Stony Brook University, Stony Brook, NY 11794-2580, USA.

A video showing $X$. fastidiosa mutant TM14 (pilY1) moving via type IV pili twitching-motility is available as supplementary data with the online version of this paper. virulent strains multiply slowly, and rarely move from the point of inoculation. In support of this claim, it has recently been noted that some hypervirulent mutants migrate faster in plants than the wild-type isolate (Guilhabert \& Kirkpatrick, 2005). The mechanism by which the bacteria are able to move from one location to another within the xylem is not entirely understood; however, we demonstrated recently that $X$. fastidiosa is able to migrate upstream, via twitching motility, using type IV pili (Meng et al., 2005), thus providing an explanation for downward migration in the plant; it was further shown that the bacterium migrates preferentially against a fluid current, suggesting that twitching motility provides the means for long-distance intra-plant movement. Type I pili are also present at the cell poles, and are thought to be involved in the development of biofilms and cell aggregation.

Twitching motility is a means of flagellar-independent bacterial movement over moist surfaces (Mattick, 2002). Twitching is known to function in host colonization, and it occurs through extension, attachment, and then retraction, of the polar type IV pili. It has been observed mostly in saprophytic bacteria and mammalian pathogens. Such motility in plant-associated bacteria has been observed in Ralstonia solanacearum (Kang et al., 2002; Liu et al., 2001) and X. fastidiosa (Meng et al., 2005). To date, approximately 
40 genes have been identified that are involved in the biogenesis and function of type IV pili in Pseudomonas aeruginosa (Mattick, 2002), including the genes that encode the major structural protein (PilA), and those that encode the minor proteins involved in formation of the base and/or tip of the pilus, e.g. PilE, PilV, PilW, PilX, PilY1, PilY2 and FimT. A number of other proteins are required for pilus assembly and retraction, e.g. PilB, PilC, PilD, PilF, PilM, PilN, PilO, PilP, PilQ, PilT and PilU. In addition, a set of genes encodes regulatory proteins that control the production of pili, and the activity of twitching motility in response to environmental stimuli; these genes include the twocomponent sensor-regulator pair pilS/pilR, and the genes associated with a chemosensory regulatory system: pilGpilK and chpA-chpE. Studies have indicated that pilA mutants (pilin deficient) of $P$. aeruginosa are avirulent or have reduced virulence (Comolli et al., 1999; Hazlett et al., 1991), and that pilA, pilT and pilQ mutants of the phytopathogen $R$. solanacearum cause slower disease development, and less severe wilting symptoms, in tomato plants (Kang et al., 2002; Liu et al., 2001).

In addition to twitching motility, cell attachment and biofilm formation are thought to be important factors in pathogen virulence. Attachment to host surfaces by cellsurface adhesins is an essential early event in pathogenesis of many bacterial pathogens (Hélaine et al., 2005). Attachment is likely to function as the initial step in the formation of a biofilm, i.e. a community or population of micro-organisms attached to a solid surface that, as a structural unit, may provide survival advantages to the bacteria when exposed to different environments (de Souza et al., 2004). In Escherichia coli, for example, type I pili play a central role in cell attachment and biofilm formation (Blumer et al., 2005; Schilling et al., 2001). In other bacteria, type IV pili have also been shown to function in cell attachment and biofilm formation (Hélaine et al., 2005). For example, in Neisseria meningitidis and Neisseria gonorrhoeae, type IV pili facilitate bacterial attachment to human cells (Carbonnelle et al., 2005). Marques et al. (2002) demonstrated that X. fastidiosa isolates from various hosts formed biofilms on wood in an in vitro assay, and hypothesized that biofilm formation is likely to be a major virulence factor in disease caused by $X$. fast $i-$ diosa. Our recent study (Meng et al., 2005) revealed that the wild-type Temecula isolate of $X$. fastidiosa possesses both type I pili and type IV pili: type I pili play a central role in cell attachment and biofilm formation, and type IV pili mediate twitching motility on agar surfaces and against the flow of nutrient media in microfluidic chambers. A fimA mutant (type I pili deficient) was capable of moving upstream via twitching motility; however, pilB and pilQ mutants, which no longer produced type IV pili, did not exhibit a twitching phenotype. The pilB and pilQ mutants were also greatly impaired in their ability to migrate within grapevine shoots (Meng et al., 2005).

In this study, we report on the identity of several hitherto undescribed genes that are associated with the formation of type IV pili in X. fastidiosa. The association of specific genes with twitching motility, biofilm formation and cell-cell aggregation is determined.

\section{METHODS}

Bacterial isolates and culture. The wild-type isolate of $X$. fastidiosa Temecula (ATCC 700964), originally isolated from a grapevine with Pierce's disease grown in Temecula, California (Van Sluys et al., 2003), was maintained on PW (Davis et al., 1981) agar modified by

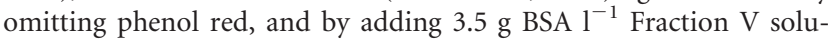
tion (A-8918; Sigma) instead of $6.0 \mathrm{~g} \mathrm{BSA}^{-1}$. Mutants were maintained on modified PW agar containing $50 \mathrm{mg}$ kanamycin $1^{-1}$. Liquid modified PW was used for preparation of electrocompetent cells, and mutants were grown in PD2 (Davis et al., 1981) for DNA isolation and subsequent sequence analyses. $X$. fastidiosa isolates and mutants were stored at $-80{ }^{\circ} \mathrm{C}$ in modified PW broth containing $7 \%$ DMSO (final concentration; Sigma).

Mutagenesis. The EZ::TN Transposome system $<$ Kan-2 $>$ kit (Epicentre) was used to generate $X$. fastidiosa mutants. Electrocompetent cells were prepared as described by Guilhabert et al. (2001). Electroporation was conducted at $2500 \mathrm{~V}, 200 \Omega$ and $25 \mu \mathrm{F}$ for $5 \mathrm{~ms}$, and the electroporated cells were plated on modified PW agar plus kanamycin. A second mutation in the fimA mutant 6E11 (Meng et al., 2005) was accomplished with the EZ::TN Transposome $<$ DHFR-1 $>$ kit (Epicentre). The electroporated cells were subsequently plated on modified PW agar plus kanamycin $\left(50 \mathrm{mg} \mathrm{l}^{-1}\right)$ and trimethoprim $\left(250 \mathrm{mg} \mathrm{l}^{-1}\right)$. Individual colonies of putative mutants were verified by PCR for Tn 5 insertions.

Screening for twitching motility mutants. Mutants were screened for twitching motility on modified PW containing $1.8 \mathrm{~g}$ BSA $1^{-1}$, and solidified with $1.2 \%$ agar. Bacteria were picked from modified PW agar with a sterile toothpick, and gently spotted onto PW agar surfaces. The plates were incubated at $28^{\circ} \mathrm{C}$ for 3 days, after which the edge morphology of the colonies was examined using a dissecting microscope (SZX12; Olympus). Colonies with a peripheral fringe were designated as twitch positive (Meng et al., 2005). Colonies lacking a peripheral fringe were checked several times to verify the twitch-minus phenotype. Some mutants were further examined microscopically in microfabricated fluidic chambers using time-lapse imaging (Meng et al., 2005).

Sequence analysis. Genomic DNA from twitching-defective mutants was digested with EcoRI, generating fragments that included the Tn5 insertion. The fragments were then cloned into the EcoRI site of pUC18. DNA sequences of disrupted genes were determined by using transposon-specific primers (provided by Epicentre) reading sequences outwards from the transposon. About 800-1000 nt were sequenced for each mutant of interest. The genomic locations of Tn5 insertions were identified using a BLAST search of the $X$. fastidiosa Temecula genome database (http://aeg.lbi.ic.unicamp.br/world/xfpd/).

Southern blotting. Confirmation of the presence of a single Tn5 insertion in mutants of interest was performed by Southern analysis. Genomic DNA isolated from twitching-defective mutants was digested with HindIII, and electrophoretically separated on $1 \%$ agarose. DNAs were alkali denatured, transferred to a hybridization transfer membrane (NEM Life Science Products), and fixed by baking at $85-90^{\circ} \mathrm{C}$ for $2 \mathrm{~h}$. Tn5 DNA was PCR amplified, purified, and labelled with DIG High Prime DNA 1 Labelling and Detection Starter kit 1 (Roche). The labelled Tn5 was used as a hybridization probe in Southern blot analysis of genomic DNAs from the mutants.

Electron microscopy. Cells were generally obtained from the periphery of 2-day-old colonies. Cells were mixed with distilled water, 
deposited on Formvar-coated grids, and subsequently stained with either phosphotungstic acid or uranyl acetate, and examined with a JEOL S-100 transmission electron microscope.

Biofilm formation and cell-cell aggregation. Cells taken from 5- to 7-day-old cultures grown on PW agar plates were suspended in liquid PD2, and adjusted to an $\mathrm{OD}_{600}$ of 0.1 . A $100 \mu \mathrm{l}$ cell suspension was added to $1 \mathrm{ml} \mathrm{PD} 2$ in polystyrene tubes, polypropylene tubes, and glass tubes, and grown at $28^{\circ} \mathrm{C}$ for 7 days, without agitation. Subsequently, the medium was stained with $100 \mu \mathrm{l} 0.1 \%$ crystal violet for $20 \mathrm{~min}$, and rinsed three times with distilled water. The presence of a biofilm was visualized as a purple ring on the tube side wall, usually at the air-medium interface (O'Toole et al., 1999). In a separate study, biofilm development was assessed on the inner surface of $250 \mathrm{ml}$ glass Erlenmeyer flasks, along with aggregation of $X$. fastidiosa cells at the bottom of the medium in the same flasks. For these observations, $300 \mu \mathrm{l}$ cell suspension, as described above, was added to $50 \mathrm{ml} \mathrm{PD} 2$. Flasks were incubated at room temperature (approximately $22^{\circ} \mathrm{C}$ ), with agitation (195 r.p.m.), for 7-10 days. Following assessment of biofilm development and cell aggregation, the contents of the flasks were removed, and the integrity of the side wall biofilm was determined by adding $50 \mathrm{ml}$ distilled water, and swirling vigorously to disperse cells that were not tightly bound to the flasks.

Growth rates. Cells from 5- to 7-day-old cultures grown on modified PW agar were suspended in modified PW broth $(3.5 \mathrm{~g}$ BSA $\left.1^{-1}\right)$, and adjusted to an $\mathrm{OD}_{600}$ of 0.1 . A $300 \mu \mathrm{l}$ cell suspension was added to $10 \mathrm{ml} \mathrm{PW}$ broth in glass culture tubes $(18 \mathrm{~cm}$ long), and incubated at $28^{\circ} \mathrm{C}$, with continuous agitation at 195 r.p.m. Cell density was determined at regular intervals by measuring $\mathrm{OD}_{600}$. The experiment was repeated twice, independently.

\section{RESULTS}

\section{Mutagenesis and screening of twitching mutants}

High transformation efficiency was achieved (up to $7.5 \times$ $10^{4}$ transformants per $\mu \mathrm{g}$ transposon DNA) when Tn5 transposomes were electroporated into $X$. fastidiosa. Transposon insertion (fragment size 700-800 bp) was detected in all mutants tested by PCR, but was not present in the wild-type $X$. fastidiosa isolate. Putative twitch-minus mutants were selected by the presence of a smooth colony margin, i.e. the absence of a twitching-associated peripheral fringe (Fig. 1) (Meng et al., 2005). Thirty twitchingdefective mutants were obtained from approximately $3000 \mathrm{Kan}^{\mathrm{R}}$ insertion mutants, seven of which are described in this report (Table 1). Southern analysis confirmed that a single insertion occurred in the genome of each mutant selected for further study (data not shown).

In addition to single insertion mutants, a second round of mutagenesis into the previously described mutant 6E11, which was deficient for type I pili, but retained type IV pili and a twitching phenotype (Meng et al., 2005), was performed to create a 'double' mutant that lacked both type I and type IV pili. Six non-twitching double mutants were obtained, one of which (DM12) is reported herein.

\section{Sequence analysis of mutants}

The DNA sequence of the regions flanking the transposon insertions of the twitching mutants revealed that in the 30 mutants the transposon had inserted into 12 different ORFs. Those in which insertions occurred in ORFs that are homologues of pilus-related genes in $P$. aeruginosa, including PD0019 (fimT, one mutant), PD0022 (pilX, one mutant), PD0023 (pilY1, two mutants), PD1693 (pilO, two mutants), and PD1928 (pilR, two mutants), were selected for further investigation. In these organisms, the disrupted genes reside in four different pilus-related gene clusters (Fig. 2). We have previously reported mutations in pilB and pilQ (Fig. 2; Meng et al., 2005). DNA sequence alignment analysis indicated high levels of identity between these genes compared with type-IV-pilus-associated genes in $P$. aeruginosa (Table 1). In the previously described mutant 6E11 (Meng et al., 2005), the transposon insertion occurred in ORF PD0062 (Fig. 2), which corresponded to the fimA gene of E. coli whose product is the major protein subunit precursor for type I pili. The second insertions in mutant 6E11

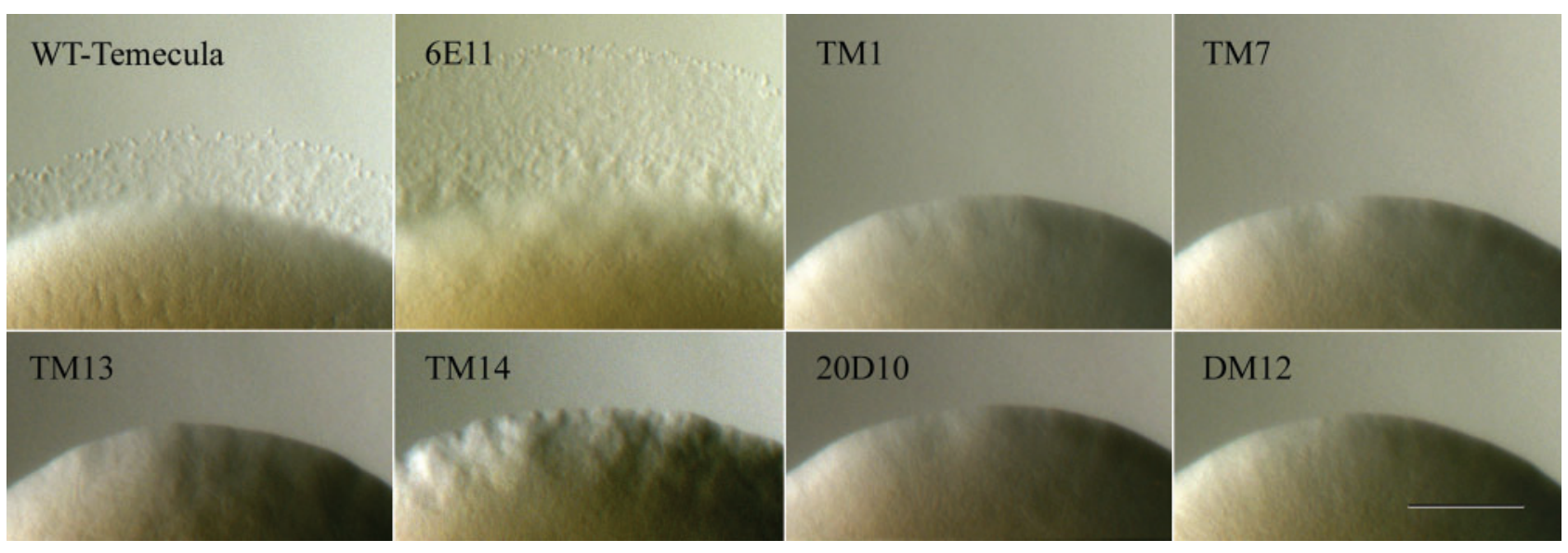

Fig. 1. Colony morphologies of wild-type $X$. fastidiosa Temecula isolate and mutants grown on a modified PW agar surface for 4 days. Scale bar, $0.1 \mathrm{~mm}$. 
Table 1. Mutants and identities of the disrupted genes in $X$. fastidiosa with their homologues in $P$. aeruginosa

\begin{tabular}{|c|c|c|c|c|}
\hline \multicolumn{2}{|c|}{ X. fastidiosa } & \multicolumn{2}{|r|}{ P. aeruginosa } & \multirow{2}{*}{$\begin{array}{l}\text { Percentage } \\
\text { identity } \ddagger\end{array}$} \\
\hline Mutant & Gene (ORF) & Gene (ORF) & Predicted gene product & \\
\hline $\mathrm{TM}^{*} 3^{*}$ & fimT (PD0019) & fim T (PA4549) & Type IV fimbrial biogenesis protein FimT & 29 \\
\hline $20 \mathrm{D} 10^{*}$ & pilX (PD0022) & pilX (PA4553) & Type IV fimbrial biogenesis protein PilX & 24 \\
\hline $\mathrm{TM} 14 \dagger$ & pilY1 (PD0023) & pilY1 (PA4554) & Type IV fimbrial biogenesis protein PilY1 & 31 \\
\hline $5 \mathrm{~A} 7^{\star}$ & pilQ (PD1691) & pilQ (PA5040) & $\begin{array}{l}\text { Type IV fimbrial biogenesis outer-membrane } \\
\text { protein. PilQ precursor }\end{array}$ & 39 \\
\hline $\mathrm{TM}^{*}$ & pilO (PD1693) & pilO (PA5042) & Type IV fimbrial biogenesis protein $\mathrm{PilO}$ & 41 \\
\hline $1 \mathrm{~A} 2^{*}$ & pilB (PD1927) & pilB (PA4526) & Type IV fimbrial biogenesis protein PilB & 56 \\
\hline $\mathrm{TM}^{\star}$ & pilR (PD1928) & pilR (PA4547) & Two-component response regulator PilR & 58 \\
\hline
\end{tabular}

${ }^{\star}$ Colony margin smooth and not fringed.

†Colony margin smooth and not fringed, occasionally crenulated.

‡Identity to $P$. aeruginosa.

occurred in PD1923 (pilC in DM11 and DM15), PD1693 (pilO in DM12), PD1671 (DM13), PD0609 (DM14) and PD0022 (pilX in DM16), respectively. Among these double mutants, DM12 (fimA/pilO) (Fig. 2) was selected for further study because it possessed neither type I nor type IV pili. The pilO gene resides in operon pilMNOPQ (Van Sluys et al., 2003), whose homologues in $P$. aeruginosa are required for type IV pilus assembly (Martin et al., 1995; Mattick, 2002).

\section{Pili}

As previously reported, $X$. fastidiosa possesses both type I pili (0.4-1.0 $\mu \mathrm{m}$ in length) and type IV pili (1.0-5.8 $\mu \mathrm{m}$ in length) (Fig. 3a; Meng et al., 2005). Transmission electron microscopy revealed that the twitch-minus mutants TM1 (pilO), TM7 (pilR), TM13 (fimT) and 20D10 (pilX) lacked type IV pili, but possessed type I pili (Fig. 3b). The fimA

(a)
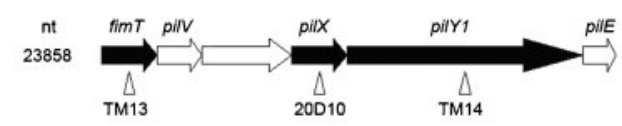

(b)
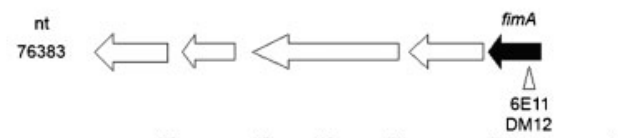

(c)

$\mathrm{nt}$
1966572
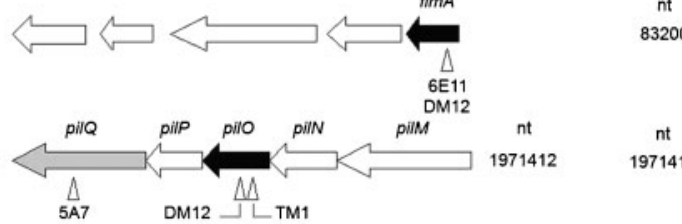

(d)
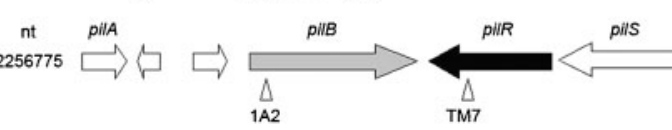

Fig. 2. Genomic organization of genes flanking Tn5 insertions in X. fastidiosa. nt, Nucleotide; black arrows, genes disrupted in this study; grey arrows, genes disrupted in a previously reported study (Meng et al., 2005). (a) Gene cluster flanking fimT, pilX and pilY1. (b) Gene cluster flanking fimA. (c). Gene cluster flanking pilO and pilQ. (d) Gene cluster flanking pilB and pilR. mutant 6E11 (Fig. 3d) was used to create the double mutant DM12, which lacked both types of pili (Fig. 3c). Both type I and type IV pili were observed in the pilY1 mutant TM14 (not shown), and they appeared similar to those observed in the wild-type isolate.

\section{Twitching motility of wild-type and mutants}

Mutants with peripheral fringe morphologies of colonies that differed from wild-type $X$. fastidiosa were selected (Fig. 1). Mutants TM1 (pilO), TM7 (pilR), TM13 (fimT) and 20D10 (pilX) exhibited smooth colony margins on both

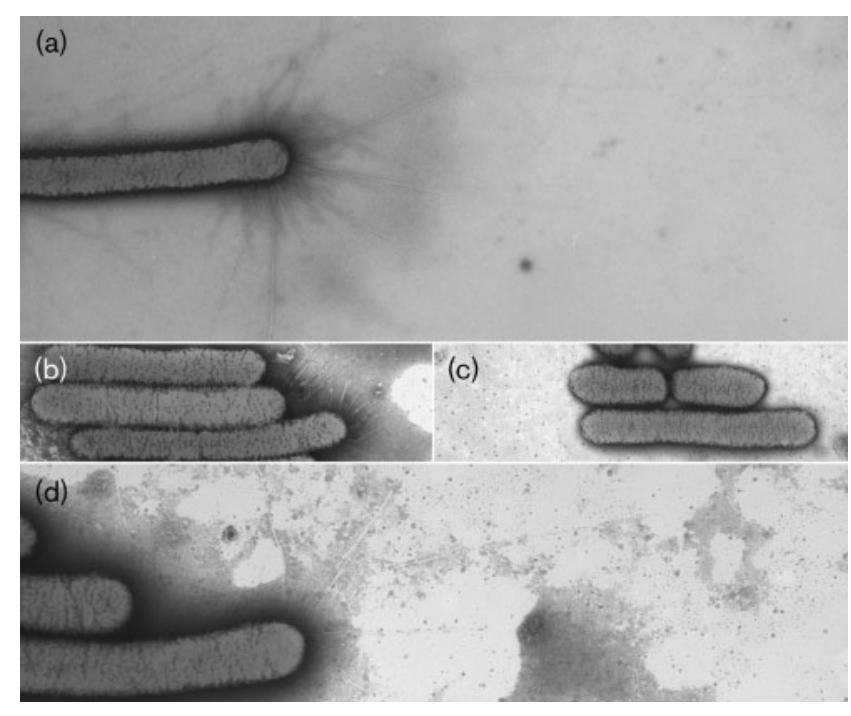

Fig. 3. Transmission electron micrographs of negatively stained cells of $X$. fastidiosa depicting type I and/or type IV pili. (a) Wild-type Temecula isolate with both short type I pili and longer type IV pili; (b) mutant TM1 with only type I pili (mutants TM7, TM13 and 20D10 had similar pilus morphologies); (c) mutant DM12 with no pili; and (d) mutant 6E11 with type IV pili only. 
PD2 (data not shown) and modified PW agar surfaces, indicating that they lacked twitching motility (Fig. 1), and were similar to the phenotype previously reported in pilB and pilQ mutants (Meng et al., 2005). Mutant TM14 (pilY1) exhibited a crenulated colony margin, suggesting reduced, but not completely impaired, twitching motility. Subsequently, time-lapse examination of this mutant showed that it retained partial motility (a video of this is available as supplementary data with the online version of this paper, and also at www.nysaes.cornell.edu/pp/faculty/ hoch/movies). Mutant 6E11 (fimA), previously reported to be twitch positive (Meng et al., 2005; also see http://www. nysaes.cornell.edu/pp/faculty/hoch/movies), exhibited a peripheral colony fringe similar to that observed in the wild-type. Further observation in this study revealed that the peripheral fringe of 6Ell colonies was nearly always wider than the fringe of the wild-type isolate, suggesting a greater rate of motility in 6E11 (Fig. 1). The double mutant DM12 ( fimA/pilO) did not exhibit a colony peripheral fringe, was incapable of twitching, and lacked both type I and type IV pili (Fig. 1).

\section{Biofilms, cell aggregates, and growth rates}

We have previously demonstrated that biofilm formation in X. fastidiosa is influenced by the presence of type I and type IV pili (Meng et al., 2005). In this study we further characterized the development of biofilms and cell-cell aggregates in newly generated pilus mutants on different surfaces and media. Since it has been observed that cells of $X$. fastidiosa Temecula attach poorly to surfaces and to each other when cultured in PW medium, PD2 was used for observations of biofilm formation. When $X$. fastidiosa wildtype and twitching-defective mutants were cultured without agitation, they formed visible biofilms on polystyrene and polypropylene surfaces; in contrast, 6E11 and DM12 exhibited significantly reduced biofilms on similar surfaces (Fig. 4). The twitch-minus mutants that lacked only type IV pili formed more robust biofilms on polystyrene and glass surfaces than the wild-type isolate; however, no differences were observed on polypropylene with these same mutants, suggesting that the surface material greatly affects attachment of the bacteria. We also tested biofilm formation by the wild-type, and TM1, 6E11 and DM12, cultured in glass Erlenmeyer flasks with continuous agitation (Fig. 5a). TM1 formed significantly more biofilm than did the wild-type isolate. This result was similar to that reported for the pilB and pilQ mutants (Meng et al., 2005). The biofilm formed by 6E11 was visibly reduced as compared to the wild-type isolate or TM1 (Fig. 5a). DM12 developed no visible biofilm on the flasks (Fig. 5a). Furthermore, it was noted that the biofilms formed by the wild-type and 6E11 were easily removed from the flask surfaces by swirling in distilled water, whereas the biofilm formed by TM1 remained intact (data not shown).

The quantity of non-attached cell aggregates at the bottom of the culture vessels was approximately inversely proportional to the amount of biofilm on the flask side walls

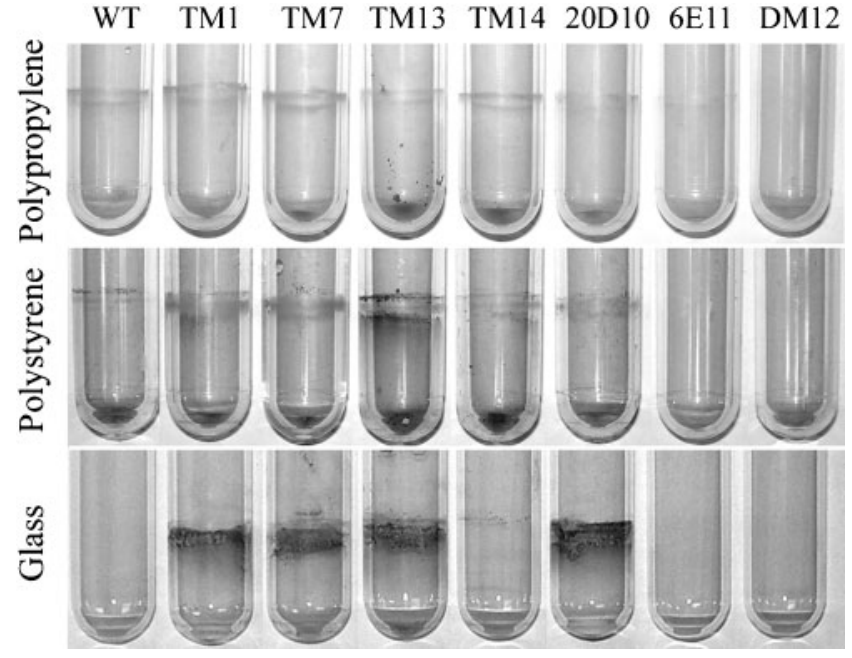

Fig. 4. Biofilm formation by $X$. fastidiosa wild-type Temecula isolate and various mutants on polypropylene, polystyrene and glass surfaces following 7 days of growth in stationary culture, and staining with crystal violet.

(Fig. 5). In addition, there were distinct differences in the size and morphologies of these aggregates. DM12 aggregates were not only numerous but small in size and lens-shaped

(b)

WT

(a)

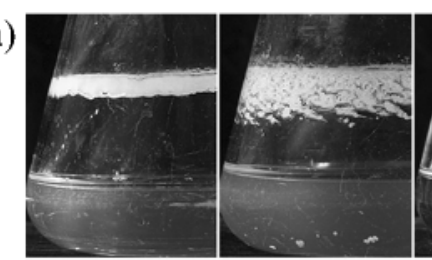

$6 \mathrm{E} 11$

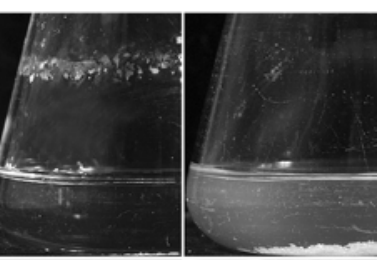

(c)
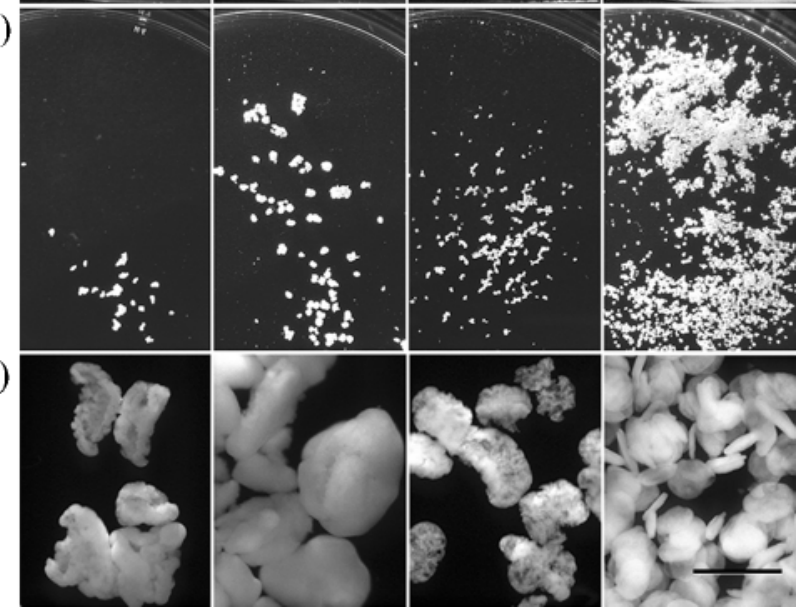

Fig. 5. Biofilm formation and cell-cell aggregation of $X$. fastidiosa wild-type Temecula isolate and various mutants following 7 days of growth in culture with agitation. (a) Biofilm on inner surface of flasks; (b) and (c) free-floating cell aggregates from the bottom of the flasks. Scale bar, $1.0 \mathrm{~mm}$. 
compared with the other mutants and the wild-type (Fig. 5). The TM1 mutant consistently produced the largest aggregates, whereas the wild-type and the 6E11 mutant, both with the longer type IV pili, produced loosely compact aggregates (Fig. 5). These results suggest that afimbrial adhesions play an important role in cell-cell aggregation. In contrast, the aggregates formed by the wild-type, TM1 and 6E11 were larger than those formed by DM12, suggesting that the presence of pili causes more cells to clump together, and form larger aggregates. Growth rates of the various mutants did not differ significantly from that of wild-type $X$. fastidiosa (not shown); therefore, the twitching phenotypes observed in the mutants were not the result of altered growth ability.

\section{DISCUSSION}

The genome of the Pierce's disease strain of $X$. fastidiosa contains at least 25 putative pil genes that are likely to be associated with type IV pilus formation and twitching motility (Van Sluys et al., 2003; Meng et al., 2005). The mutants described in the current study are associated with genes that are homologous to the pil genes in $P$. aeruginosa. In $P$. aeruginosa, FimT, PilX and PilY1 are minor proteins that are predicted to be associated with the base and/or tip of the type IV pilus (Mattick, 2002). In N. meningitidis, PilX has been reported to be essential for bacterial aggregation and pilus-mediated attachment to human cells (Hélaine et al., 2005). PilY1 in P. aeruginosa is associated with the cell membrane, and is required for the development of extracellular pili and twitching (Alm et al., 1996). PilY1 is a homologue of PilC2, which is a pilus tip adhesion protein in N. gonorrhoeae (Alm et al., 1996). We have observed type IV pili in the pilY1 mutant; this is to be expected if the gene encodes the tip adhesion protein. The mutation would presumably affect the ability of the pilus to attach to the agar surface, thereby affecting twitching motility. The pilY1 mutant displayed a crenulated colony periphery, and was shown to twitch less effectively than the wild-type.

The pilO gene is located in a putative operon consisting of PD1691-PD1695 (pilMNOPQ). The predicted pilO gene product shares $41 \%$ identity with $\mathrm{PilO}$ of $P$. aeruginosa (Table 1). In P. aeruginosa, this operon is required for pilus assembly (Martin et al., 1995). A pilQ mutant in X. fastidiosa failed to produce type IV pili, and had a twitch-minus phenotype (Meng et al., 2005). Another pil gene cluster, PD1922-PD1928, contains pilB and pilR, whose predicted protein products share 56 and $58 \%$ identities, respectively, with the homologous PilB and PilR in $P$. aeruginosa (Table 1). The gene cluster in $P$. aeruginosa is thought to function in type IV pilus biogenesis and twitching motility (Mattick, 2002; Hobbs et al., 1993). PilS and PilR belong to the family of two-component transcriptional regulatory systems that have been described in many bacterial species (Hobbs et al., 1993). PilS is a predicted sensory protein that, when stimulated by the appropriate environmental signals, activates PilR through kinase activity; PilR then regulates transcription of pilA (Hobbs et al., 1993). We have shown that the pilR mutant lacks type IV pili, and does not twitch, which may result from lack of pilA expression.

An interesting characteristic of $X$. fastidiosa is that it possesses both type I and type IV pili, predominantly at one cell pole. The fimA gene is located in the cluster PD0058PD0062 (Van Sluys et al., 2003). Homologues of this gene cluster in E. coli are required for biofilm formation, and FimA is the major subunit for type I pili (Blumer et al., 2005; Schilling et al., 2001). The predicted protein product of fimA in $X$. fastidiosa shares $23.1 \%$ identity with the homologue FimA in E. coli K-12. The fimA mutant of X. fastidiosa was unable to produce type I pili, but was able to produce type IV pili, and maintain twitching motility. Feil et al. (2003) disrupted fimA and fimH in X. fastidiosa, and observed that fimbria (i.e. pilus) size and number, as well as cell aggregation and aggregate size, were reduced in both fim $A$ and fim $H$ mutants. Interestingly, the mutants remained pathogenic in grapevines.

\section{Pili and twitching motility}

Type-IV-pilus-mediated twitching motility has been documented for many bacteria (Hobbs et al., 1993; Liu et al., 2001; Meng et al., 2005). Without type IV pili, X. fastidiosa cells were unable to twitch, whereas the fimA mutant remained twitch positive. We observed, based on colony peripheral fringe width, that the twitching motility of the fimA mutant (6E11) was enhanced compared with the wildtype; this provides further evidence that type I pili are not required for twitching motility. The enhanced twitching motility of the fimA mutant suggests that the presence of type I pili may partially restrict cell movement.

\section{Biofilm formation and cell-cell aggregation}

Type I pili of $X$. fastidiosa play fundamental roles in biofilm formation and cell-cell aggregation. The twitch-minus mutants TM1, TM7, TM13 and 20D10, which lack type IV pili, produced more abundant biofilms and larger cell aggregates than the wild-type isolate or any of the other mutants (Figs 4 and 5). Mutants that possessed only type IV pili (6E11) exhibited less pronounced biofilms, and the mutant with no pili (DM12) did not produce an easily discernible biofilm. For DM12, afimbrial cell surface adhesins may have led to the formation of the numerous small aggregates. This result is consistent with a report (Guilhabert \& Kirkpatrick, 2005) that haemagglutinins mediate cell-cell aggregation in X. fastidiosa. When the gene $h x f A$ (PD2118) was disrupted, the ability to form aggregates in liquid culture was impaired. The haemagglutinin adhesions produced by Erwinia chrysanthemi have also been shown to contribute to aggregation (Rojas et al., 2002). When either or both type I and type IV pili are present, larger aggregates are formed, suggesting a role for pili (Figs 4 and 5). It is not surprising that some biofilm formation occurred in the mutant with only type IV pili (6E11), since these pili are involved in twitching motility, which is a 
mechanism that requires cell attachment to substrata. Similar roles of attachment for type IV pili have been shown in $P$. aeruginosa and other bacteria (O'Toole \& Kolter, 1998; Scheuerpflug et al., 1999). In addition, the ability to attach to host surfaces has been shown to be essential for virulence in other systems (Merz \& So, 2000; Mattick, 2002). We show that type IV pili also play an important role in surface attachment and biofilm formation. Our results suggest that type IV pili play a role in biofilm formation; however, the biofilm density seems to be greatly influenced by the presence of type I pili.

The results of this study improve our understanding of how $X$. fastidiosa pili influence twitching motility, biofilm formation and cell-cell aggregation. The roles of the identified pilus genes in movement and colonization within plants, and ultimately disease, will be further investigated. The elucidation of genetic mechanisms associated with virulence of $X$. fastidiosa may be exploited for the development of novel controls for Pierce's disease.

\section{ACKNOWLEDGEMENTS}

We extend our thanks to Steve Lindow (Dept of Plant and Microbial Biology, University of California, Berkeley, CA, USA) and Bruce Kirkpatrick (Dept of Plant Pathology, University of California, Davis, CA, USA) for providing the $X$. fastidiosa wild-type Temecula isolate, and for suggestions regarding $X$. fastidiosa biology during the course of this investigation. Funding for this project was provided, in part, by grants from the Nanobiotechnology Center (NBTC), an STC Program of the National Science Foundation under Agreement No. ECS9876771, and from the USDA/CSREES administered through the University of California Pierce's Disease Research Grants Program. This work was also performed, in part, at the Cornell Nanofabrication Facility (a member of the National Nanofabrication Users Network), which is supported by the National Science Foundation under Grant ECS-9731293, its users, Cornell University, and Industrial Affiliates.

\section{REFERENCES}

Alm, R. A., Hallinan, J. P., Watson, A. A. \& Mattick, J. S. (1996). Fimbrial biogenesis genes of Pseudomonas aeruginosa: pilW and pilX increase the similarity of type 4 fimbriae to the GSP proteinsecretion systems and pilY1 encodes a gonococcal PilC homologue. Mol Microbiol 22, 161-173.

Blumer, C., Kleefeld, A., Lehnen, D., Heintz, M., Dobrindt, U., Nagy, G., Michaelis, K., Emody, L., Polen, T. \& other authors (2005). Regulation of type 1 fimbriae synthesis and biofilm formation by the transcriptional regulator LrhA of Escherichia coli. Microbiology 151, 3287-3298.

Carbonnelle, E., Hélaine, S., Prouvensier, L., Nassif, X. \& Pelicic, V. (2005). Type IV pilus biogenesis in Neisseria meningitidis: PilW is involved in a step occurring after pilus assembly, essential for fibre stability and function. Mol Microbiol 55, 54-64.

Comolli, J. C., Hauser, A. R., Waite, L., Whitchurch, C. B., Mattick, J. S. \& Engel, J. N. (1999). Pseudomonas aeruginosa gene products PilT and PilU are required for cytotoxicity in vitro and virulence in a mouse model of acute pneumonia. Infect Immun 67, 3625-3630.

Davis, M. J., French, W. J. \& Schaad, N. W. (1981). Axenic culture of the bacteria associated with phony disease of peach and plum leaf scald. Curr Microbiol 6, 309-314. de Souza, A. A., Takita, M. A., Coletta-Filho, H. D., Caldana, C., Yanai, G. M., Muto, N. H., de Oliveira, R. C., Nunes, L. R. \& Machado, M. A. (2004). Gene expression profile of the plant pathogen Xylella fastidiosa during biofilm formation in vitro. FEMS Microbiol Lett 237, 341-353.

Feil, H., Feil, W. S., Detter, J. C., Purcell, A. H. \& Lindow, S. E. (2003). Site-directed disruption of the fimA and fimF genes of Xylella fastidiosa. Phytopathology 93, 675-682.

Guilhabert, M. R. \& Kirkpatrick, B. C. (2005). Identification of Xylella fastidiosa antivirulence genes: hemagglutinin adhesins contribute to $X$. fastidiosa biofilm maturation and colonization and attenuate virulence. Mol Plant Microbe Interact 18, 856-868.

Guilhabert, M. R., Hoffman, L. M., Mills, D. A. \& Kirkpatrick, B. C. (2001). Transposon mutagenesis of Xylella fastidiosa by electroporation of Tn5 synaptic complexes. Mol Plant Microbe Interact 14, 701-706.

Hazlett, L. D., Moon, M. M., Singh, A., Berk, R. S. \& Rudner, X. L. (1991). Analysis of adhesion, piliation, protease production and ocular infectivity of several $P$. aeruginosa strains. Curr Eye Res 10, 351-362.

Hélaine, S., Carbonnelle, E., Prouvensier, L., Beretti, J.-L., Nassif, X. \& Pelicic, V. (2005). PilX, a pilus-associated protein essential for bacterial aggregation, is a key to pilus-facilitated attachment of Neisseria meningitidis to human cells. Mol Microbiol 55, 65-77.

Hill, B. L. \& Purcell, A. H. (1995). Acquisition and retention of Xylella fastidiosa by an efficient vector, Graphocephala atropunctata. Phtopathology 85, 209-212.

Hobbs, M., Collie, E. S. R., Free, P. D., Livingston, S. P. \& Mattick, J. S. (1993). PilS and PilR, a two-component transcriptional regulatory system controlling expression of type 4 fimbriae in Pseudomonas aeruginosa. Mol Microbiol 7, 669-682.

Hopkins, D. L. (1989). Xylem-limited bacterial pathogen of plants. Annu Rev Phytopathol 27, 271-290.

Kang, Y., Liu, H., Genin, S., Schell, M. A. \& Denny, T. P. (2002). Ralstonia solanacearum requires type 4 pili to adhere to multiple surfaces and for natural transformation and virulence. Mol Microbiol 46, 427-437.

Liu, H., Kang, Y., Genin, S., Schell, M. A. \& Denny, T. P. (2001). Twitching motility of Ralstonia solanacearum requires a type IV pilus system. Microbiology 147, 3215-3229.

Marques, L. L. R., Ceri, H., Manfio, G. P., Reid, D. M. \& Olson, M. E. (2002). Characterization of biofilm formation by Xylella fastidiosa in vitro. Plant Dis 86, 633-638.

Martin, P. R., Watson, A. A., McCaul, T. F. \& Mattick, J. S. (1995). Characterization of a five-gene cluster required for the biogenesis of type 4 fimbriae in Pseudomonas aeruginosa. Mol Microbiol 16, 497-508.

Mattick, J. S. (2002). Type IV pili and twitching motility. Annu Rev Microbiol 56, 289-314.

Meng, Y., Li, Y., Galvani, C. D., Hao, G., Turner, J. N., Burr, T. J. \& Hoch, H. C. (2005). Upstream migration of Xylella fastidiosa via pilus-driven twitching motility. J Bacteriol 187, 5560-5567.

Merz, A. J. \& So, M. (2000). Interactions of pathogenic Neisseriae with epithelial cell membranes. Annu Rev Cell Dev Biol 16, 423-457.

O’Toole, G. A. \& Kolter, R. (1998). Flagellar and twitching motility are necessary for Pseudomonas aeruginosa biofilm development. Mol Microbiol 30, 295-304.

O'Toole, G. A., Pratt, L. A., Watnick, P. I., Newman, D. K., Weaver, V. B. \& Kolter, R. (1999). Genetic approaches to study of biofilms. Methods Enzymol 310, 91-109.

Purcell, A. H. (1996). Fastidious xylem-limited bacterial plant pathogens. Annu Rev Phytopathol 34, 131-151. 
Purcell, A. H. (1997). Xylella fastidiosa, a regional problem or global threat? J Plant Pathology 79, 99-105.

Rojas, C. M., Jam, J. H., Deng, W., Doyle, J. J. \& Collmer, A. (2002). HecA, a member of a class of adhesions produced by diverse pathogenic bacteria, contributes to the attachment, aggregation, epidermal cell killing, and virulence phenotypes of Erwinia chrysanthemi EC16 on Nicotiana clevelandii seedlings. Proc Natl Acad Sci U S A 99, 13142-13147.

Scheuerpflug, I., Rudel, T., Ryll, R., Pandit, J. \& Meyer, T. F. (1999). Roles of PilC and PilE proteins in pilus-mediated adherence of Neisseria gonorrhoeae and Neisseria meningitidis to human erythrocytes and endothelial and epithelial cells. Infect Immun 67, 834-843.

Schilling, J. D., Mulvey, M. A. \& Hultgren, S. J. (2001). Structure and function of Escherichia coli type I pili: new insight into the pathogenesis of urinary tract infections. J Infect Dis 183, S36-40.

Tubajika, K. M., Civerolo, E. L., Ciomperlik, M. A., Luvisi, D. A. \& Hashim, J. M. (2004). Analysis of the spatial patterns of Pierce's disease incidence in the lower San Joaquin Valley in California. Phytopathology 94, 1136-1144.

Van Sluys, M. A., de Oliveira, M. C., Monteiro-Vitorello, C. B., Miyaki, C. Y., Furlan, L. R., Camargo, L. E. A., da Silva, A. C. R., Moon, D. H., Takita, M. A. \& other authors (2003). Comparative analyses of the complete genome sequences of Pierce's disease and citrus variegated chlorosis strains of Xylella fastidiosa. J Bacteriol 185, 1018-1026.

Edited by: M. F. Hynes 